\title{
More attentional focusing through binaural beats: evidence from the global-local task
}

\author{
Lorenza S. Colzato $^{1} \cdot$ Hayley Barone ${ }^{1} \cdot$ Roberta Sellaro $^{1} \cdot$ Bernhard Hommel $^{1}$
}

Received: 27 July 2015/ Accepted: 13 November 2015/Published online: 26 November 2015

(c) The Author(s) 2015. This article is published with open access at Springerlink.com

\begin{abstract}
A recent study showed that binaural beats have an impact on the efficiency of allocating attention over time. We were interested to see whether this impact affects attentional focusing or, even further, the top-down control over irrelevant information. Healthy adults listened to gamma-frequency $(40 \mathrm{~Hz})$ binaural beats, which are assumed to increase attentional concentration, or a constant tone of $340 \mathrm{~Hz}$ (control condition) for $3 \mathrm{~min}$ before and during a global-local task. While the size of the congruency effect (indicating the failure to suppress task-irrelevant information) was unaffected by the binaural beats, the global-precedence effect (reflecting attentional focusing) was considerably smaller after gamma-frequency binaural beats than after the control condition. Our findings suggest that high-frequency binaural beats bias the individual attentional processing style towards a reduced spotlight of attention.
\end{abstract}

\section{Introduction}

When two beats of slightly different frequency (for instance 300 and $340 \mathrm{~Hz}$ ) are presented separately to the left and right ears, the hearer detects a single beat that differs in amplitude at a frequency equal to the frequency difference between the two beats $(40 \mathrm{~Hz})$; a perceptual illusion known as the binaural auditory beat. While the

Lorenza S. Colzato

colzato@fsw.leidenuniv.nl

1 Cognitive Psychology Unit, Institute for Psychological Research and Leiden Institute for Brain and Cognition, Leiden University, Wassenaarseweg 52, 2333, AK, Leiden, The Netherlands neural mechanisms underlying this illusion are still unclear, very recent studies have shown that beat stimulation significantly affects functional brain connectivity (Gao et al., 2014) and modulates intracranial power and phase synchronization (Becher et al., 2015). These findings support the idea that the neural phase locking elicited by binaural beats can influence ongoing cognitive processing (Karino et al., 2006; see, Chaieb et al., 2015, for a recent review on the effect of binaural beats on cognition and mood). Lowfrequency binaural beats are associated with mental relaxation and high frequency beats with alertness and attentional concentration (Vernon, 2009; Turow \& Lane, 2011). This suggests that high-frequency beats might facilitate attentional control, which would fit with the observation that high-frequency neurofeedback training over the frontal cortex improves attentional efficiency (Keizer, Verment, \& Hommel, 2010).

Very recently Reedijk, Bolders, Colzato, and Hommel (2015) have shown that binaural auditory beats affect how people control and monitor their visual attention. Participants listened to binaural beats while performing an attentional blink (AB) task, which assesses the efficiency of allocating attention over time. The size of the $\mathrm{AB}$ was considerably reduced by the binaural beats at least in some participants, which suggests that beats have a specific impact on how people allocate their attention over time. However, it is not yet clear how binaural beats affect attentional allocation over space. A well-known task that taps into this issue is the global-local task developed by Navon (1977). This task assesses how fast people can process global versus local characteristics of hierarchically constructed visual stimuli (e.g., larger shapes made of smaller shapes). Participants are typically confronted with a global stimulus (e.g., large rectangle) that is made of smaller shapes (the local stimuli), and the relationship between global and local stimuli can be 
congruent (e.g., a large rectangle composed of small rectangles) or incongruent (a large rectangle made of small squares). Typically, this task gives rise to the "global precedence" effect (i.e., performance is better when responding to global than to local features), which implies that global features can be processed faster than local features. Even though some models suppose that global and local level information is selected from associated spatial frequency channels (Robertson \& Lamb, 1991) or by binding level information to feature-like representations of the hierarchical structure (Hübner \& Volberg, 2005; Volberg \& Hübner, 2004), global precedence is generally assumed to indicate a bias towards a large, comprehensive attentional focus, while attending to local features is considered to require more attentional effort.

There are reasons to assume that binaural beats might affect the size or scope of the attentional focus. Evidence for a role of individual differences in attentional control in the global-local task comes from Dale and Arnell (2010, 2015), who found a negative correlation between global precedence and $\mathrm{AB}$ magnitude: people who showed a smaller global precedence effect (i.e., a relatively stronger disposition towards processing local information) showed a greater $\mathrm{AB}$ magnitude. These observations suggest that individuals can exert control over the allocation of attention when processing targets. Interestingly, binaural beats might impact attention in a global-local task in two, not necessarily mutually exclusive ways.

First, processing global information is commonly assumed to require a broader, more spatially distributed focus of attention, while processing local information is assumed to rely on a smaller, more tightly controlled focus (Navon, 1977, 1981; Kimchi, 1992; Kimchi \& Palmer, 1982). As high frequency beats arguably promote alertness and attentional concentration (Vernon, 2009; Turow \& Lane, 2011), one might expect that listening to gammafrequency $(40 \mathrm{~Hz})$ binaural beats is associated with a smaller global precedence effect than listening to a constant tone of $340 \mathrm{~Hz}$ (control condition). Indeed, if gammafrequency binaural beats support the processing of local features, this should reduce the difference between performance on global and on local features. If this is the case, we would expect an interaction between the instructed target level (global vs. local) and the kind of beats (gammafrequency vs. control). Theoretically, such an interaction would indicate a relatively direct impact of binaural beats on the focus or distribution of visual attention.

Second, the global-local task generates conflict by providing irrelevant information that indicates an alternative response. As pointed out previously, global-local tasks often employ congruent and incongruent stimuli, so as to prevent strategies that use information from one level to predict information from the other. Most importantly for our purpose, performance is better in congruent than in incongruent trials (Navon, 1977). This implies that adopting a global or local task set does not prevent the processing of information related to the other task, which can be taken to indicate a task or goal conflict (Kiesel et al., 2010). If we assume that high frequency beats promote alertness and attentional concentration (Vernon, 2009; Turow \& Lane, 2011), we would predict that congruency effects are smaller when listening to gamma-frequency beats than while listening to a constant tone. If this is the case, we would expect an interaction between congruency and the kind of beats (gamma-frequency vs. control), with a smaller impact of congruency with gamma-frequency beats than with a constant tone. Theoretically, such an interaction would suggest a rather general impact of binaural beats on cognitive control, rather than a more specific impact on visual attention. Given that our previous study (Reedijk et al., 2015) has shown that the effect of binaural beats on $\mathrm{AB}$ magnitude was limited to the gamma-frequency binaural beats, we focused our investigation on the high-, but not low-frequency binaural beats.

\section{Method}

\section{Participants}

Thirty-six students (22 female, 14 male; aged 18-28 years old) from Leiden University participated in this study in exchange for course credit or pay. All had normal or corrected-to-normal sight and hearing. Participants were selected individually using the Mini International Neuropsychiatric Interview (MINI; Sheehan et al., 1998). The MINI is a well-established brief diagnostic tool in clinical, drug and stress research that screens for several psychiatric disorders and drug use (Sheehan et al., 1998; Colzato, Kool \& Hommel, 2008, Colzato et al., 2011). Randomly, 18 participants (10 female, 8 male) were exposed to gammafrequency $(40 \mathrm{~Hz})$ binaural beats session and 18 (12 female, 6 male) to a control condition (a constant tone of $340 \mathrm{~Hz}$ ) session. Written informed consent was obtained from all subjects; the protocol and the remuneration arrangements of 5 euro were approved by the local ethical committee (Leiden University, Institute for Psychological Research).

\section{Global-local task}

The task was adopted from Colzato, van den Wildenberg and Hommel (2008) and Steenbergen et al. (2015). The experiment was controlled by a Windows-operated computer attached to a Philips $17^{\prime \prime}$ monitor. Participants were seated at a viewing distance of $57 \mathrm{~cm}$ from the monitor 
(screen resolution: $1024 \times 768$; refresh rate: $100 \mathrm{~Hz}$ ). Responses were made by pressing the " $Z$ " or "/" of the QWERTY computer keyboard with the left and right index finger, respectively. The target stimuli consisted of hierarchical geometric figures, namely, larger (global) rectangles $\left(1.5^{\circ} \times 6.4^{\circ}\right)$ or squares $\left(3.2^{\circ} \times 3.2^{\circ}\right)$ composed of 16 smaller (local) rectangles $\left(0.3^{\circ} \times 1.5^{\circ}\right)$ or squares $\left(0.7^{\circ} \times 0.7^{\circ}\right)$. The space between the local elements of a stimulus was $0.1^{\circ}$ both horizontally and vertically. In each trial, participants were presented with one of the four possible target stimuli: a rectangle consisting of smaller rectangles or squares, or a square consisting of smaller rectangles or squares. They had to alternate between responding to the local and to the global dimension of the target stimuli every four trials. The rectangle or square was associated with a spatially assigned response button that was pressed with either the left (" $\mathrm{z}$ " from computer keyboard) or right ("/" from computer keyboard) index finger; the stimulus-response mapping was randomized across participants. Target stimuli were presented either in the upper or in the lower part of the screen, depending on the to-be-attended level. Target stimuli presented in the upper part of the screen signaled to respond to the local (global) level, whereas those presented in the lower part of the screen signaled to respond to the global (local) level. The target position-local/global rule assignment varied randomly across participants. The to-be-attended level (global or local) was primed by a cue appearing 400-600 ms before the target stimulus (see Fig. 1). Cues that related to the global (local) dimension consisted of a big (small) square, presented at one side of the target stimulus, and a big (small) rectangle, presented at the other side of the target stimulus. The "local" and "global" cues were the same size as the global and local stimuli, and their left/right location corresponded to the position of the required response. Cues and target stimuli were presented in red on a white background, and both remained on the screen until a response was given or $2500 \mathrm{~ms}$ had passed. The interval between response and presentation of the next cue was 900-1100 ms (see Fig. 1).

The task consisted of a single experimental block of 160 trials, in which the four possible target stimuli were presented with equal probabilities. Therefore, participants performed on a total of 80 global trials and 80 local trials. Half of the trials were congruent (a large square consisting of smaller squares or a large rectangle consisting of smaller rectangles) and the other half of the trials were incongruent (a large square consisting of smaller rectangles or a large rectangle consisting of smaller squares). Moreover, of the resulting 160 trials, 39 included a task/level switch and 120 (not considering the very first trial) a task/level repetition. The experimental block was preceded by two training blocks of 50 trials each, in which the dimension to be attended (global or local) was constant across all trials within that block. Training block order was counterbalanced between participants, meaning that half of the
Fig. 1 Sequence of events of a local trial followed by a global trial. Larger (global) rectangles/ squares consisted of smaller (local) rectangles or squares. The congruent trial presents a bigger shape composed of similar smaller shapes (e.g., a large square consisting of smaller squares), the incongruent trial presents a bigger shape composed of different smaller shapes (e.g., a large rectangle consisting of smaller squares)

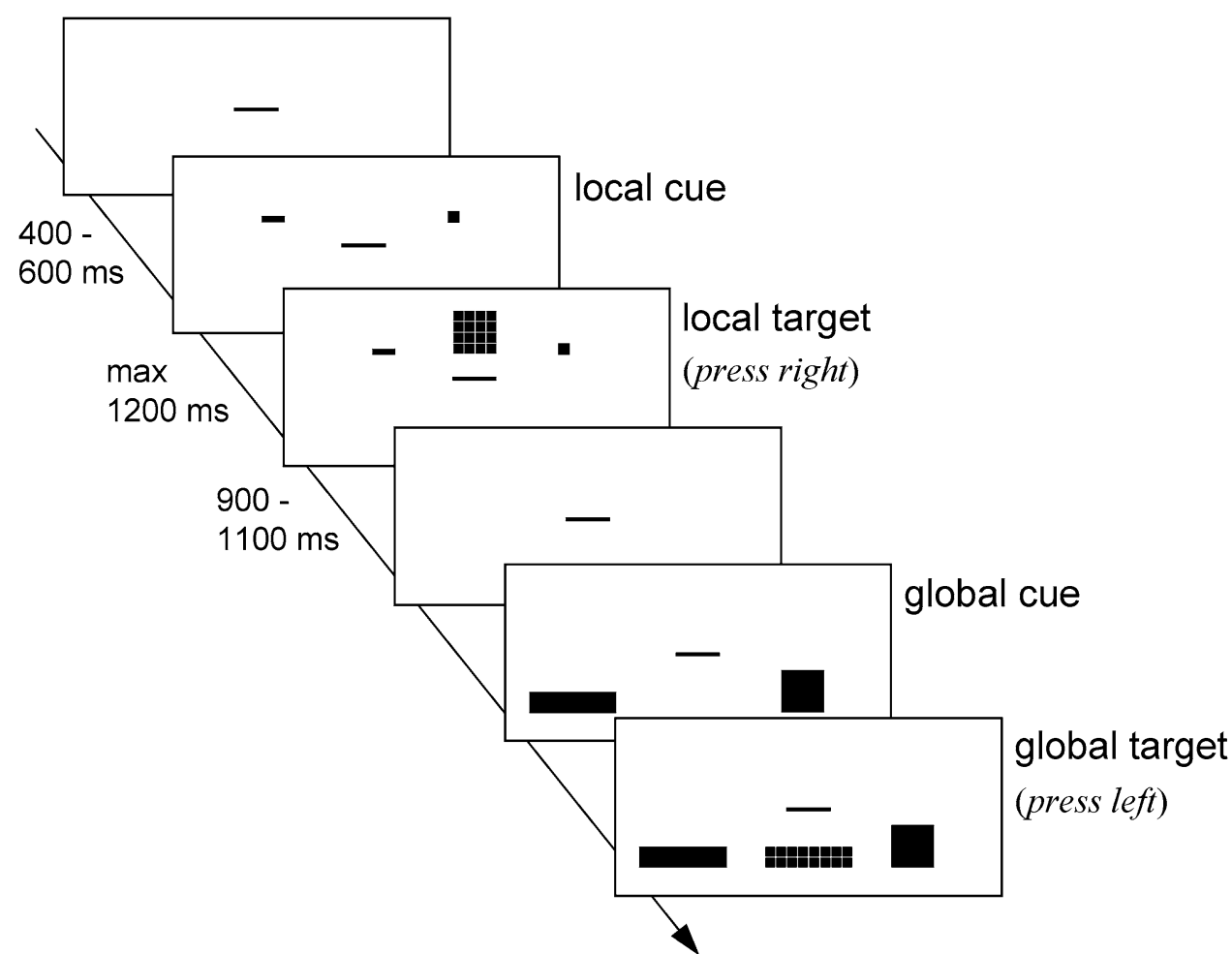


participants started with the "local block," the other half with the "global block."

\section{Procedure}

Participants were invited individually to the laboratory. In both sessions, upon arrival, they were asked to rate their mood on a $9 \times 9$ Pleasure $\times$ Arousal grid (Russell, Weis, \& Mendelsohn, 1989) with values ranging from -4 to 4 . Thus, the scale provides a score that indicates the location of the participant's affective state within a two-dimensional space defined by hedonic tone and activation. Subsequently, participants listened to gamma-frequency $(40 \mathrm{~Hz})$ binaural beats or a constant tone of $340 \mathrm{~Hz}$ (control condition), all embedded in white noise to enhance clarity of the beats (Oster, 1973), for 3 min before and during the global-local task (training and experimental blocks). Binaural beats were presented through in-ear headphones (Etymotic Research ER-4B microPro), which provide $35 \mathrm{~dB}$ noise attenuation. Both binaural beat conditions were based on a $340 \mathrm{~Hz}$ carrier tone, which was used as the constant tone in the control condition. After the globallocal task, participants rated their mood for the second time. After these measurements, the experimental session was ended and participants were paid, debriefed and dismissed.

\section{Statistical analysis}

Mean RTs and proportions of errors were analyzed by means of ANOVAs using target level (global vs. local), the congruency between the stimuli on the two levels (congruent vs. incongruent), and task switch (i.e., same vs. different target level as in previous trial: task repetition vs. alternation) as within-subjects factor and group (gamma vs. control) as between-subject factor. Mood was analyzed by means of a repeated-measures analysis of variance (ANOVA) with effect of time (first vs. second measurement) as within-subjects factor and group (gamma vs. control) as between-subject factor. A significance level of $p<.05$ was adopted for all statistical tests.

\section{Results}

\section{Global-local task}

The reaction time analysis showed five significant sources of variance, see Table 1. First, the effect of target level, $F(1,34)=80.97, p<.0001, \mathrm{MSE}=1879.08, \eta_{p}^{2}=0.70$, reflecting the well-known global precedence effect (Navon, 1977), that is, faster responses to globally than locally defined targets (421 vs. $467 \mathrm{~ms}$ ). Second, the effect of congruency, $F(1,34)=31.70, p<.0001, \mathrm{MSE}=1502.73$, $\eta_{p}^{2}=0.48$, reflecting interference of the irrelevant target level, as indicated by a faster RT on congruent as compared to incongruent trials ( 431 vs. $457 \mathrm{~ms}$ ). Third, the effect of switching, $F(1,34)=27.59, p<.0001, \mathrm{MSE}=7429.21$, $\eta_{p}^{2}=0.45$, which revealed that repeating the task allowed for faster responding than switching between target levels (418 vs. $471 \mathrm{~ms})$. Fourth, the interaction of task switch and congruency, $\quad F(1,34)=4.22, \quad p<.05, \quad \mathrm{MSE}=1459.05$, $\eta_{p}^{2}=0.11$, indicated that the congruency effect was larger when target levels were switching (35 ms) than when they were repeated $(17 \mathrm{~ms})$. Most importantly for our purposes, target level interacted with group, $F(1,34)=4.21, p<.05$, MSE $=1879.08, \eta_{p}^{2}=0.11$ (see Fig. 2 ): the size of the global precedence effect was significantly smaller in the gamma group (36 ms) than in the control group (57 ms). Last, both switch and congruency did not interact significantly with group, $F(1,34)=1.61, p>.05, \quad \mathrm{MSE}=$ $7429.21, \eta_{p}^{2}=0.04$ and $F<1$, respectively. Given that conventional null-hypothesis significance testing (NHST) cannot be used to provide evidence in favor of the null hypothesis $\left(H_{0}\right)$, we calculated the Bayesian (posterior) probability associated with the occurrence of the null hypothesis $\left[p\left(H_{0} \mid D\right)\right]$ to validate the absence of any interaction between the factors group and congruency (crucial for our second hypothesis). To this end we used the method proposed by Wagenmakers (2007) and Masson (2011). This method uses Bayesian information criteria (BIC), calculated using a simple transformation of sum-of-squares values generated by the standard ANOVA, to estimate the Bayes factor and generate $p\left(H_{0} \mid D\right)$, assuming a "unit information prior" (for further details, see Kass \& Wasserman, 1995; see also Jarosz \& Wiley, 2014).

The $p\left(H_{0} \mid D\right)$ provides the exact probability of the occurrence of $H_{0}$. The analysis revealed that the $p\left(H_{0} l-\right.$ $D$ ) was 0.84 , hence, on the basis of the guidelines proposed by Raftery (1995), represents positive evidence in favor of $H_{0}$.

The analysis of the error rates revealed only a main effect of congruency, $F(1,34)=70.31, \quad p<.0001$, $\mathrm{MSE}=53.59, \eta_{p}^{2}=0.67$, reflecting interference from the irrelevant target level, as indicated by a smaller proportion of errors in congruent than incongruent trials (2.9 vs. $10.2 \%)$.

\section{Mood and arousal}

As expected, the ANOVA performed on participants' mean mood and arousal rating revealed a significant interaction between time and group only for arousal, $F(1,34)=4.29$, $p<.05, \mathrm{MSE}=0.93, \eta_{p}^{2}=0.11$, but not $\operatorname{mood}, F \leq 1$. LSD Fisher post hoc analyses revealed that, for the control group, arousal levels were comparable across the two 
Table 1 Mean reaction times (RT; in $\mathrm{ms}$ ) and errors (in \%) for each condition in the globallocal task-switching paradigm as a function of and group (control vs. gamma) are displayed

\begin{tabular}{|c|c|c|c|c|}
\hline \multirow[t]{2}{*}{ Group } & \multicolumn{2}{|l|}{ Control } & \multicolumn{2}{|l|}{ Gamma } \\
\hline & Mean RT & Mean error & Mean RT & Mean error \\
\hline Switch & $495(22.2)$ & $8.2(1.3)$ & $447(22.2)$ & $5.6(1.3)$ \\
\hline Repetition & $428(11.2)$ & $6.2(0.9)$ & $407(11.2)$ & $6.1(0.9)$ \\
\hline Switch cost & $67 \mathrm{~ms}$ & & $40 \mathrm{~ms}$ & \\
\hline Local target & $490(16.1)$ & $8.8(1.5)$ & $445(16.1)$ & $6.3(1.5)$ \\
\hline Global target & $433(16.8)$ & $5.6(0.9)$ & $409(16.8)$ & $5.5(0.9)$ \\
\hline Global precedence effect & $57 \mathrm{~ms}$ & & $36 \mathrm{~ms}$ & \\
\hline Incongruent & $476(18.3)$ & $11.4(1.4)$ & $439(18.3)$ & $8.9(1.4)$ \\
\hline Congruent & $448(14.1)$ & $3.0(0.8)$ & $415(14.1)$ & $2.8(0.8)$ \\
\hline Congruency effect & $28 \mathrm{~ms}$ & & $24 \mathrm{~ms}$ & \\
\hline
\end{tabular}

Standard errors are shown in parentheses

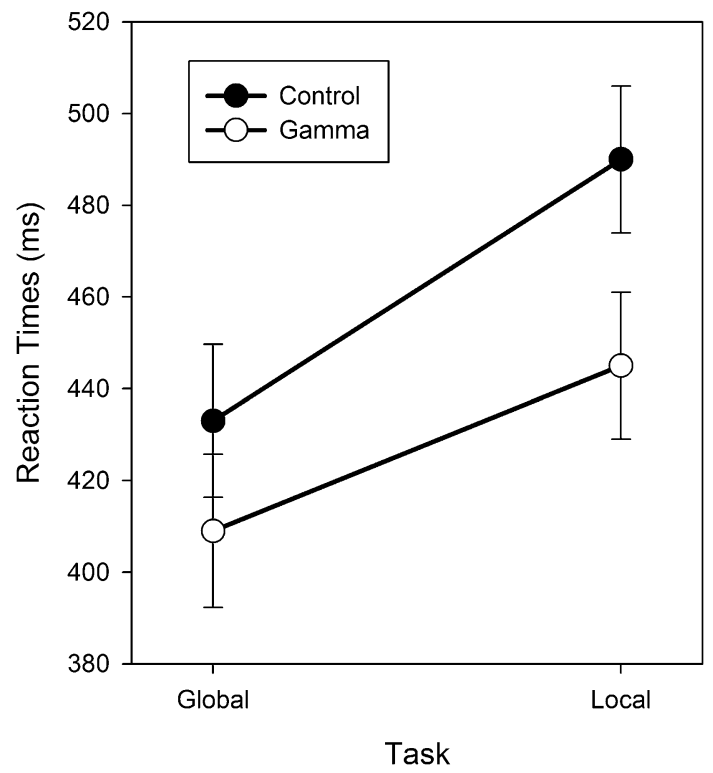

Fig. 2 Mean reaction times (ms) as a function of group (gamma vs. control) and target level (global vs. local). Error bars represent standard errors

measurements (arousal levels were 0.5 and 0.3 , for the first and second measurement, respectively, $p=.61$ ). In contrast, for the gamma group, a significant difference between the two time points indicated an increase from the first to the second measurement ( 0.3 vs. $1.1, p=.02$ ), suggesting that our manipulation worked as expected.

\section{Discussion}

The findings of our study are straightforward. First, corroborated by Bayesian inference, there was no indication that the congruency effect would be affected by binaural beats. If we consider that congruency reflects crosstalk from a currently irrelevant task or stimulus dimension (Kiesel et al., 2010), our observation indicates that gammafrequency binaural beats do not lead participants/people to further engage in the suppression of currently irrelevant information in working memory. Second, however, highfrequency binaural beats did have a significant impact on the global precedence effect: the precedence effect became smaller, suggesting that visual attention became more focused than in the control condition.

A possible explanation of how high-frequency binaural beats might enhance attentional focus could mean that listening to gamma binaural beats entrains gamma band activity in the brain. Increased activity in the gamma frequency band is typically associated with greater attentional investment (Keizer et al., 2010; Rieder, Rahm, Williams \& Kaiser, 2011), which in turn is associated with a deeper attentional blink (Olivers \& Nieuwenhuis, 2006) and reduced global precedence effect (Dale \& Arnell 2010, 2015). One way to test this possible connection would be to entrain gamma activity through mechanisms/techniques other than binaural beats, which should have the same effect. If successful, this might provide an interesting avenue for supporting attentional control abilities in clinical populations suffering from attentional disorders, such as attention-deficit-/hyperactivity disorder.

As pointed out in the introduction, it has been also proposed that global and local level information is selected by binding level information to feature-like representations of the hierarchical structure (Hübner \& Volberg, 2005; Volberg \& Hübner, 2004). This idea is particularly intriguing given that previous studies have suggested that the underlying neural underpinnings of binding features in the visual domain is supported by transient increases in synchronization in the gamma frequency range (Colzato et al., 2004, 2005). Accordingly, it may be that high-frequency binaural beats enhance binding processes within 
the visual cortex by entraining synchronization frequencies in the gamma band (Engel \& Singer, 2001).

It seems interesting to contrast the observations of the present study with those of Reedijk et al. (2015) on the one hand and of Dale and Arnell $(2010,2015)$ on the other. While Reedijk et al. (2015) found that binaural beats eliminated the $\mathrm{AB}$ at least in some individuals, we found that binaural beats are reducing the global precedence effect. This would seem to suggest a positive correlation between the global precedence effect and the $\mathrm{AB}$, which does not fit with Dale and Arnell's $(2010,2015)$ records of negative correlations. This apparent discrepancy might suggest that the mechanisms underlying the impact of binaural beats on the $\mathrm{AB}$ and those underlying the impact of binaural beats on the global precedence effect are not the same. For instance, it might be that the precedence effect is affected through gamma-entrainment while the $A B$ is affected through providing a temporal reference frame. Given that the $\mathrm{AB}$ can be reduced by optimal preparation of neural control states (Gross et al., 2006), a reduction of temporal uncertainty should result in a smaller effect. If the presentation of binaural beats provides a regular background that helps to predict the temporal onset of targets in the $\mathrm{AB}$ task, reduced $\mathrm{AB}$ should indeed be observed. For the time being however, this must remain speculation.

Our investigation used a between-subjects design to avoid possible practice effects on task performance. One cannot rule out that the differences we found in the globallocal task are actually due to pre-existing group differences in cognitive-control styles rather than due to binaural beats exposure. Therefore, follow-up studies should determine whether our findings can be replicated in a within-subject comparison using different version of the global-local task and other tasks suited for investigating the attentional focusing, such as a flanker paradigm with varying distances between target and flanker.

In any case, our findings bring convergent evidence to the idea that binaural beats can affect and enhance cognition (Lane et al., 1998; Reedijk et al., 2013, 2015). While this is encouraging for the purpose of behavioral optimization in healthy and clinical populations, future studies should investigate how long-lasting binaural beats-induced biases of attentional control are. In sum, this is the first study to demonstrate that binaural beats induce an individual processing style that modulates visual attention. As we have argued, beats in the gamma range achieve this effect by increasing attentional focusing rather than by suppressing currently irrelevant tasks and task-related information.

Acknowledgments The research of L.S. Colzato is supported by a Vidi Grant (\#452-12-001) of the Netherlands Organization for Scientific Research (NWO).
Open Access This article is distributed under the terms of the Creative Commons Attribution 4.0 International License (http://crea tivecommons.org/licenses/by/4.0/), which permits unrestricted use, distribution, and reproduction in any medium, provided you give appropriate credit to the original author(s) and the source, provide a link to the Creative Commons license, and indicate if changes were made.

\section{References}

Becher, A. K., Höhne, M., Axmacher, N., Chaieb, L., Elger, C. E., \& Fell, J. (2015). Intracranial electroencephalography power and phase synchronization changes during monaural and binaural beat stimulation. European Journal of Neuroscience, 41(2), 254-263.

Chaieb, L., Wilpert, E. C., Reber, T. P., \& Fell, J. (2015). Auditory beat stimulation and its effects on cognition and mood states. Frontiers in Psychiatry, 6, 70.

Colzato, L. S., Erasmus, V., \& Hommel, B. (2004). Moderate alcohol consumption in humans impairs feature binding in visual perception but not across perception and action. Neuroscience Letters, 360, 103-105.

Colzato, L. S., Fagioli, S., Erasmus, V., \& Hommel, B. (2005). Caffeine, but not nicotine enhances visual feature binding. European Journal of Neuroscience, 21, 591-595.

Colzato, L. S., Kool, W., \& Hommel, B. (2008a). Stress modulation of visuomotor binding. Neuropsychologia, 46(5), 1542-1548.

Colzato, L. S., Ruiz, M. J., van den Wildenberg, W. P., \& Hommel, B. (2011). Khat use is associated with impaired working memory and cognitive flexibility. PLoS One, 6(6), e20602.

Colzato, L. S., van den Wildenberg, W. P. M., \& Hommel, B. (2008b). Losing the big picture: How religion may control visual attention. PLoS One, 3, e3679.

Dale, G., \& Arnell, K. M. (2010). Individual differences in dispositional focus of attention predict attentional blink magnitude. Attention, Perception, and Psychophysics, 72(3), 602-606.

Dale, G., \& Arnell, K. M. (2015). Multiple measures of dispositional global/local bias predict attentional blink magnitude. Psychological Research, 79, 534-547.

Engel, A. K., \& Singer, W. (2001). Temporal binding and the neural correlates of sensory awareness. Trends in Cognitive Science, 5 , $16-25$.

Gao, X., Cao, H., Ming, D., Qi, H., Wang, X., Wang, X., \& Zhou, P. (2014). Analysis of EEG activity in response to binaural beats with different frequencies. International Journal of Psychophysiology, 94(3), 399-406.

Gross, J., Schmitz, F., Schnitzler, I., Kessler, K., Shapiro, K., Hommel, B., \& Schnitzler, A. (2006). Anticipatory control of long-range phase synchronization. European Journal of Neuroscience, 24, 2057-2060.

Hübner, R., \& Volberg, G. (2005). The integration of object levels and their content: A theory of global/local processing and related hemispheric differences. Journal of Experimental Psychology: Human Perception and Performance, 31(3), 520.

Jarosz, A. F., \& Wiley, J. (2014). What are the odds? A practical guide to computing and reporting Bayes Factors. The Journal of Problem Solving, 7, 2. doi:10.7771/1932-6246.1167.

Karino, S., Yumoto, M., Itoh, K., Uno, A., Yamakawa, K., Sekimoto, S., \& Kaga, K. (2006). Neuromagnetic responses to binaural beat in human cerebral cortex. Journal of Neurophysiology, 96, 1927-1938.

Kass, R. E., \& Wasserman, L. (1995). A reference Bayesian test for nested hypotheses and its relationship to the Schwarz criterion. 
Journal of American Statistical Association, 90, 928-934. doi:10.1080/01621459.1995.10476592.

Keizer, A. W., Verment, R., \& Hommel, B. (2010). Enhancing cognitive control through neurofeedback: A role of gamma-band activity in managing episodic retrieval. Neuroimage, 49, 3404-3413.

Kiesel, A., Steinhauser, M., Wendt, M., Falkenstein, M., Jost, K., Phillip, A., \& Koch, I. (2010). Control and interference in task switching-A review. Psychological Bulletin, 136, 849-874.

Kimchi, R. (1992). Primacy of wholistic processing and global/local paradigm: A critical review. Psychological Bulletin, 112(1), 24-38.

Kimchi, R., \& Palmer, S. E. (1982). Form and texture in hierarchically constructed patterns. Journal of Experimental Psychology: Human Perception and Performance, 8(4), 521-535.

Lane, J. D., Kasian, S. J., Owens, J. E., \& Marsh, G. R. (1998). Binaural auditory beats affect vigilance, performance and mood. Physiology and Behavior, 63, 249-252.

Masson, M. E. J. (2011). A tutorial on a practical Bayesian alternative to null hypothesis significance testing. Behavior Research Methods, 43, 679-690.

Navon, D. (1977). Forest before trees: The precedence of global features in visual perception. Cognitive Psychology, 9, 353-383.

Navon, D. (1981). The forest revisited: More on global precedence. Psychological Research, 43(1), 1-32.

Olivers, C. N. L., \& Nieuwenhuis, S. (2006). The beneficial effects of additional task load, positive affect, and instruction on the attentional blink. Journal of Experimental Psychology: Human Perception and Performance, 32, 364-379.

Oster, G. (1973). Auditory beats in the brain. Scientific American, 229, 94-102.

Raftery, A. E. (1995). Bayesian model selection in social research. In P. V. Marsden (Ed.), Sociological methodology (pp. 111-196). Oxford: Blackwells.

Reedijk, S. A., Bolders, A., Colzato, L. S., \& Hommel, B. (2015). Eliminating the attentional blink through binaural beats: A case for tailored cognitive enhancement. Frontiers in Psychiatry, 6, 82 .
Reedijk, S. A., Bolders, A., \& Hommel, B. (2013). The impact of binaural beats on creativity. Frontiers in Human Neuroscience, 7, 786.

Rieder, M. K., Rahm, B., Williams, J. D., \& Kaiser, J. (2011). Human gamma-band activity and behavior. International Journal of Psychophysiology, 79, 39-48.

Robertson, L. C., \& Lamb, M. R. (1991). Neuropsychological contributions to theories of part/whole organization. Cognitive Psychology, 23(2), 299-330.

Russel, J. A., Weiss, A., \& Mendelsohn, G. A. (1989). Affect grid: A single-item scale of pleasure and arousal. Journal of Personality and Social Psychology, 57, 493-502.

Sheehan, D. V., Lecrubier, Y., Sheehan, K. H., Amorim, P., Janavs, J., Weiller, \& Dunbar, G. C. (1998). The Mini-International Neuropsychiatric Interview (MINI): The development and validation of a structured diagnostic psychiatric interview for DSM-IV and ICD-10. Journal of Clinical Psychiatry, 59, 22-23.

Steenbergen, L., Sellaro, R., de Rover, M., Hommel, B., \& Colzato, L. S. (2015). No role of beta receptors in cognitive flexibility: Evidence from a task-switching paradigm in a randomized controlled trial. Neuroscience, 295, 237-242.

Turow, G., \& Lane, J. D. (2011). Binaural beat stimulation: Altering vigilance and mood states. In J. Berger \& G. Turow (Eds.), Music, science, and the rhythmic brain: Cultural and clinical implications (pp. 122-136). New York: Routledge.

Vernon, D. (2009). Human potential: Exploring techniques used to enhance human performance. London: Routledge.

Volberg, G., \& Hübner, R. (2004). On the role of response conflicts and stimulus position for hemispheric differences in global/local processing: An ERP study. Neuropsychologia, 42(13), 1805-1813.

Wagenmakers, E.-J. (2007). A practical solution to the pervasive problems of $p$ values. Psychonomic Bulletin and Review, 14, 779-804. 